\title{
How do adult humans compare with New Caledonian crows in tool selectivity?
}

\author{
Francisco J. Silva and Kathleen M. Silva \\ University of Redlands, Redlands, California
}

\begin{abstract}
We examined humans' tool selections on stick-and-tube tasks similar to those used to study crows' and other avian species' physical cognition. In Experiment 1, the participants selected a stick from a set of 10 to retrieve a candy placed in a horizontal tube. Although the stick that was selected depended on the distance to the candy, the participants generally did not select a stick whose length was the same as the candy's distance from the open end of the tube nor did they select the longest stick in the set - two strategies that have been reported in crows. In Experiments 2 and 3, we used variations of the stick-and-tube task to determine what factors in addition to the candy's distance influenced the participants' selections. The results showed that tool selection depended on the stimulus context (i.e., the number and lengths of the alternative tools).
\end{abstract}

When presented with a set of 10 stick tools that varied in length, two captive New Caledonian crows (Corvus moneduloides) most often attempted to retrieve food in a horizontal pipe by selecting the longest stick in the set and, less often, a stick whose length was exactly the same as (i.e., matched) the distance over which the food had to be retrieved (Chappell \& Kacelnik, 2002). The crows rarely selected a tool too short to retrieve the food. These results suggest that New Caledonian crows' understanding of physical causality includes the ability to identify the distance over which a desired object has to be retrieved, to identify a tool whose length matches or exceeds this distance, and then to use this tool to retrieve the object.

As potentially important as these results are-for this was the first time that this sort of tool selectivity was shown in a nonprimate species (Chappell \& Kacelnik, 2002) - there is some question about their generality. For example, 2 freeliving New Caledonian crows tested in a naturalistic setting generally selected, as their first and usually unsuccessful response, twigs or leaf stems whose length did not match the depth of a hole (deep or shallow) from which they had to retrieve food (Hunt, Rutledge, \& Gray, 2006). Similarly, using woodpecker finches (Cactospiza pallida) in a laboratory setting, Tebbich and Bshary (2004) found "no indication that any of the five birds tried to match tool length with the position of the food item in the tube" and "no significant correlation between location of food and the length of the first tool chosen" (p. 693). Three of the 5 finches generally selected longer-than-necessary sticks, though not always the longest stick in the set, to retrieve the food.

Commenting on their birds' behavior vis-à-vis Chappell and Kacelnik's (2002) results, both Hunt et al. (2006) and Tebbich and Bshary (2004) found little reason for crows or woodpecker finches to select tools whose lengths matched the distance over which a desired item had to be retrieved. Hunt et al. argued that there is no obvious ecological reason for crows to engage in this behavior in their natural environment, because crows usually position the nonworking ends of their tools along one side of their heads, which means that a portion of the tool is not inserted into a hole. Held in this manner, a tool would be ineffective if a crow selected a tool whose length matched the distance over which the food had to be retrieved. By selecting a longer tool, crows can adjust how they hold it and can use it in holes of various depths. Thus, selecting a longer tool is likely to be a more successful strategy than selecting one whose length matches the (normally unseen) depth of a hole with food. Similarly, Tebbich and Bshary argued that erring on the side of selecting a longer stick also makes sense for woodpecker finches, because there is little cost to selecting a longer tool but considerable cost to choosing a twig that is too short. In the former case, the bird need only break off a piece of the twig or hold it differently; in the latter case, the bird has to search for a new tool.

In sum, although Chappell and Kacelnik's (2002) crows selected at above chance levels a stick whose length matched the distance over which a piece of food had to be retrieved and the longest stick in the set, Hunt et al.'s (2006) crows and Tebbich and Bshary's (2004) finches selected neither of these sticks reliably. Although it is difficult to explain these different sets of results because there were many differences among the studies, there is no doubt that New Caledonian crows choose tools "of either the appropriate or greater length" (Emery \& Clayton, 2009, p. 27).

Our general goal in the present study was to examine how adult humans performed on tool-use tasks used to examine corvids' and other avian species' physical cognition. In the absence of "comparative work with other seemingly

F. J. Silva, francisco_silva@redlands.edu 
intelligent animals, notably humans and other primates" (Bluff, Weir, Rutz, Wimpenny, \& Kacelnik, 2007, p. 20), it is difficult to answer the question of whether corvids' tool use is more aptly described as a species-specific adaptation to procuring food or as evidence of their physical intelligence (Bluff et al., 2007; Emery, 2004; Emery \& Clayton, 2009). Using adult humans as subjects allows us to examine tool use and selectivity while assuming with some degree of certainty that a participant's behavior is not caused by the activation of species-typical reward-related sensitivities and actions (e.g., for some animals, solving a "detour problem," in which it is necessary to push a food reward farther away before it can be obtained, may be difficult if these animals are predisposed to engage in actions that bring food nearer), cognitive deficits (e.g., inability to understand causal constructs such as gravity, transfer of force, and connectivity), or imposing too great a cognitive load (e.g., requiring the participant to attend to, and remember, too many aspects of the problem and steps in potential solutions). None of these sorts of variables are likely to control adult humans' behavior on the simple problems used to study tool selectivity in crows, monkeys, apes, and other nonhuman animals. In addition, we can ask people to explain why they selected a particular tool, which is not possible with animals. To the extent that there is continuity among species' physical intelligence and in the controlling variables, these reasons may in some instances help researchers interpret animals' tool use (e.g., Silva, Page, \& Silva, 2005).

\section{EXPERIMENT 1}

We had two major goals in Experiment 1. Our first was to see how adult humans performed on a task similar to the one used by Chappell and Kacelnik (2002) to study tool selectivity in crows, and by Tebbich and Bshary (2004) to study this same behavior in woodpecker finches. Would humans select the longest stick tool, select a stick whose length matched the distance over which an object had to be retrieved, or employ some other strategy? Selecting the longest stick is a "safe" alternative, in that it is almost certain to work and requires relatively little cognitive effort in terms of foreseeing how it will be held and used, guessing the distance of the object from the open end of the tube, and evaluating whether a particular tool is long enough to reach the object. Alternatively, participants might select a tool whose length matches the distance over which the object has to be retrieved. But one might reasonably ask why rational humans would select a stick whose length was exactly the same as the distance over which an object had to be retrieved? As Hunt et al. (2006) and Tebbich and Bshary noted, selecting a "matching stick" is likely to be an ineffective strategy. However, there are at least two reasons why selecting a stick whose length matches an object's distance is not implausible. First, Chappell and Kacelnik's crows did it at above chance levels. Adult humans sometimes behave like nonhuman animals on tasks used to study these animals' physical cognition (e.g., Silva et al., 2005; Silva, Silva, Cover, Leslie, \& Rubalcaba, 2008). Second, and related to the first point, when it comes to selecting tools and solving problems of physical cognition, "many humans [do] not make rational choices based on a clear understanding of physical causality" (Emery \& Clayton, 2009, p. 33).

Our second major goal was to identify the working lengths of the sticks selected by the participants. Borrowing Chappell and Kacelnik's (2002) terminology, a stick's working length is the length of a stick minus the portion held in a person's hand. This dependent measure might help determine whether participants, in cases where the length of the stick they selected was not the same as the object's distance, might be matching the working length of the stick to the object's distance. Also, knowing the average working length of the sticks guided our selection of the stick tools used in Experiments 2 and 3.

\section{Method}

Participants. Twenty-six undergraduate students attending a liberal arts college in southern California volunteered for the study and received partial course credit for their involvement.

Materials. Ten $0.25-\mathrm{cm}$-diameter wooden dowels, ranging in length from 8 to $26 \mathrm{~cm}$ in increments of $2 \mathrm{~cm}$, served as tools. Participants used one of the sticks to retrieve a candy (an M\&M) from a transparent tube (4-cm diameter; $30 \mathrm{~cm}$ long) closed at one end. The tube was secured to the surface of a table. The experimenter placed the candy randomly at either 8 or $16 \mathrm{~cm}$ from the open end of the tube, which sometimes faced the left side of the table and sometimes the right. The sticks were laid flat on the table, centered and perpendicular to the tube. Each stick was separated from any adjacent stick by $3 \mathrm{~cm}$ and arranged so that the bottom ends of the sticks were aligned (see Figure 1). The position of any individual stick varied randomly between participants.

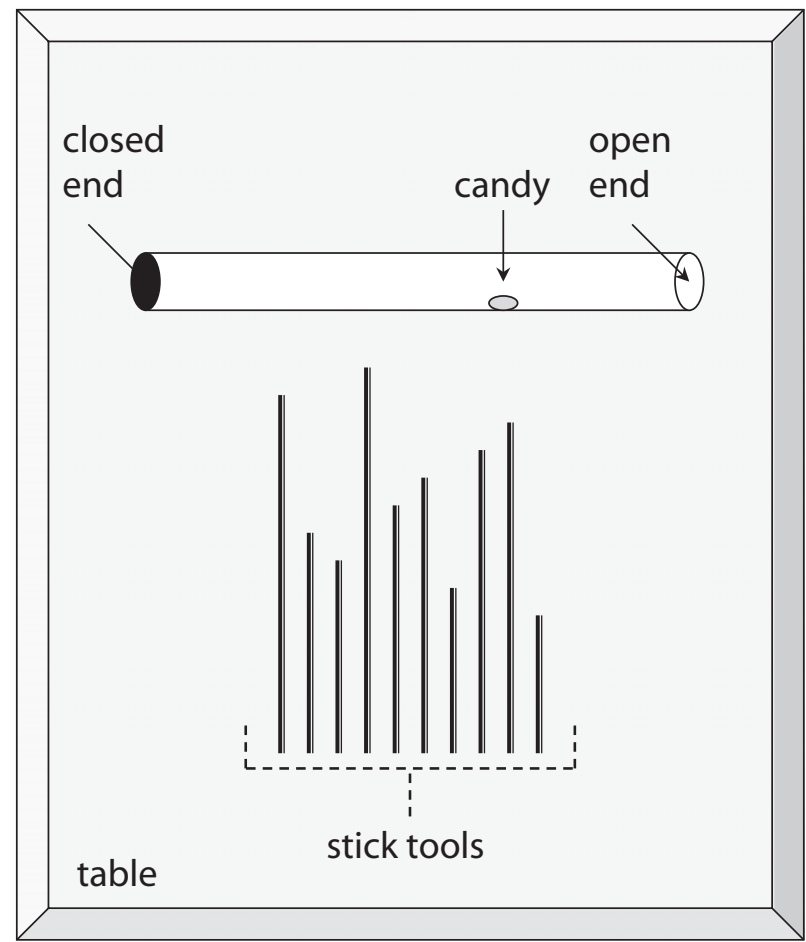

Participant stood here.

Figure 1. A schematic of the 10-stick task used in Experiment 1 (not drawn to scale). The display of the tools was randomized across participants. The distance of the food from the open end of the tube was either 8 or $16 \mathrm{~cm}$. 
Procedure. The participants were tested individually. A trial began with the experimenter asking a participant to enter the experimental room and to stand in front of the materials while the experimenter read the instructions. The experimenter told the participants they were participating in an experiment about how people use tools to solve problems, and that they had one attempt to use one of the sticks to retrieve the candy from the tube. The experimenter then instructed the participants to select a stick and to hold it as if they were ready to make their attempt. When a participant was ready, the experimenter measured the distance between the working tip of the stick (i.e., the tip that would contact the candy) and the tip of the participant's finger that was closest to the working tip. This distance was the working length of the stick. Finally, just before the participants attempted to retrieve the candy, the experimenter asked them to explain why they selected that particular stick. For all analyses, $\alpha<.05$ was used to determine statistical significance.

\section{Results and Discussion}

Figure 2 shows the mean lengths and working lengths of the sticks selected by the participants when the candy was 8 or $16 \mathrm{~cm}$ from the open end of the tube. For the 8 -cm distance, the mean length of the stick selected was $15.5 \mathrm{~cm}(S D=5.4 \mathrm{~cm})$; for the $16-\mathrm{cm}$ distance, the mean stick length was $22.6 \mathrm{~cm}(S D=2.6)$. The mean working lengths of these sticks were $11.7 \mathrm{~cm}(S D=3.4 \mathrm{~cm})$ and $19.5 \mathrm{~cm}(S D=2.8 \mathrm{~cm})$ for the 8 - and $16-\mathrm{cm}$ distances, respectively.

These results show that the participants selected a stick on the basis of the candy's distance. When the candy was $8 \mathrm{~cm}$ from the open end of the tube, the participants selected a shorter stick than when the candy was at $16 \mathrm{~cm}$ [independent-samples $t$ test: $t(24)=4.27, p<.01$ ] As holding a stick tool requires, for both distances, the working lengths of the sticks were shorter than their actual lengths. Moreover, the portion of the stick held in a participant's hand was approximately the same for each group, 3.8 and $3.1 \mathrm{~cm}$ for the people in the 8 - and $16-\mathrm{cm}$ groups, respectively [independent-samples $t$ test: $t(24)=$ $0.61, p=.55]$. Thus, regardless of the length of the stick selected, the portion held in a participant's hand was about the same.
But the results also showed that neither the mean length of the sticks nor their working lengths matched the distance over which the candy had to be retrieved. Both the actual and working lengths of the sticks were longer than the candy's distance [8-cm distance, single-sample $t$ tests: $t \mathrm{~s}(12)>3.94, p \mathrm{~s}<.01 ; 16-\mathrm{cm}$ distance, single-sample $t$ tests: $t \mathrm{~s}(12)>4.56, p \mathrm{~s}<.01]$.

Although the graph in Figure 2 shows that the participants did not generally select the longest stick or the matching stick, we can check whether these sticks were selected more or less often than expected by chance. They were not. No one in either group selected a stick whose length matched the candy's distance from the open end of the tube [binomial parameters for both the 8 - and $16-\mathrm{cm}$ distances: $n=13 ; k=0 ; p=.1 ; P$ of 0 out of $13=.25]$. Only 1 person in the 8 -cm group [binomial parameters: $n=13 ; k=1 ; p=.1 ; P$ of 1 or fewer out of $13=.62]$ and 3 people in the 16-cm group [binomial parameters: $n=$ $13 ; k=3 ; p=.1 ; P$ of 3 or more out of $13=.13]$ selected the longest stick.

In sum, the participants' general strategy was to select a tool longer than the distance over which it had to do its work. Unlike some crows (e.g., Chappell \& Kacelnik, 2002), the participants generally did not select a tool whose length or working length matched the distance over which a desired object had to be retrieved, nor did they select the longest tool in the set. Why the people in Experiment 1 behaved differently from the crows in Chappell and Kacelnik's study cannot be answered. When asked to explain their selection, most people said something about the length of a tool and its relation to usability (e.g., "It [the stick selected] was enough to reach [the candy], but not too long, which is harder to manipulate"; or, "It was the right length. There was enough room on the end to maneuver it comfortably and for it not to be too cumbersome").

That there was a difference in the mean length of the sticks selected for retrieving the candy at the 8 - and $16-\mathrm{cm}$ distances is evidence that, at the very least, the object's distance controlled the participants' selections. This result

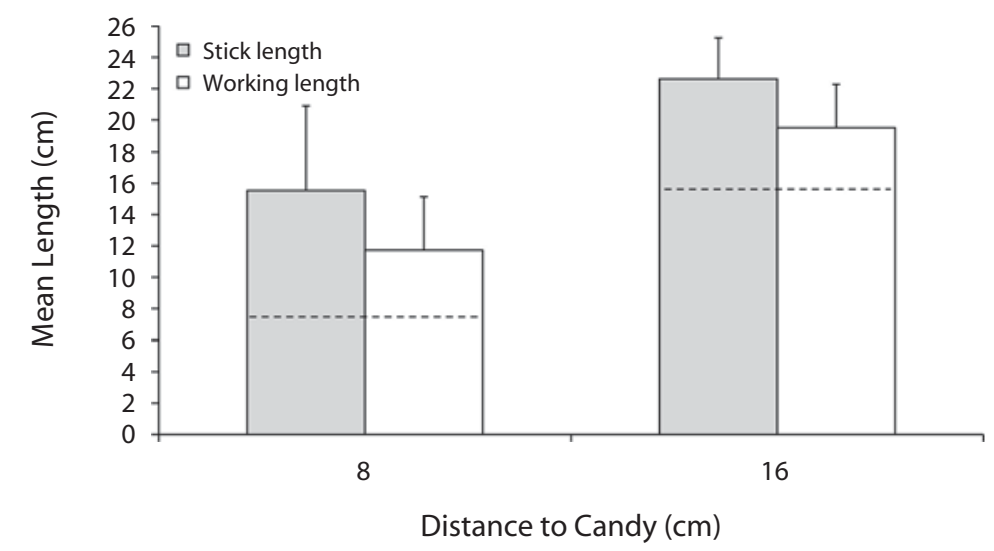

Figure 2. Mean length of the stick tools (gray bars) selected by participants in Experiment 1 when the candy was 8 or $16 \mathrm{~cm}$ from the open end of the tube. The average working length of these sticks (white bars) is also shown. The error bars show standard deviations. The dashed lines across the bars show the distance of the candy from the open end of the tube. 
contrasts with Hunt et al.'s (2006) observation that freeliving crows' initial tool selections were not related to the depth of the hole containing the food. What remains to be answered is what, in addition to the candy's distance, might have influenced the participants' selections. We address this issue in Experiments 2 and 3.

\section{EXPERIMENT 2}

The results of Experiment 1 showed that people generally selected a stick tool longer than the distance over which the desired object had to be retrieved; however, they did not select the longest stick in the set. In Experiment 2, we sought to examine the relationship between a stick's working length and the distance of the candy from the open end of the tube. We chose to focus on the working lengths of the sticks because the results of Experiment 1 showed that a stick's working length did not deviate much from the candy's distance. Recall that the mean working lengths of the sticks selected were 11.7 and $19.5 \mathrm{~cm}$ when the candy was 8 and $16 \mathrm{~cm}$ away, respectively, from the open end of the tube. For several participants, the working lengths of the sticks they selected were within a centimeter of the candy's distance. Might the participants' selections in Experiment 1 illustrate attempts to match the working length of a stick to the distance of the candy, coupled with error in judging distances and lengths?

To answer this question, we simplified the task used in Experiment 1. Because we now knew which sticks people were likely to select and their corresponding working lengths, we reduced the 10-alternative arrangements used in Experiment 1 to 2-alternative arrangements. With the candy $8 \mathrm{~cm}$ from the open end of the tube, we pitted the stick whose working length most closely matched the distance of the candy (i.e., the $12-\mathrm{cm}$ stick) against the sticks that Chappell and Kacelnik's (2002) crows selected above chance levels - that is, a stick whose actual length matched the desired object's distance (i.e., the 8-cm stick) and the longest stick in the set used in Experiment 1 (i.e., the 26-cm stick). To complete the pairwise comparisons, we also pitted a stick whose length matched the candy's distance from the open end of the tube (i.e., the $8-\mathrm{cm}$ stick) against the longest stick in the set (i.e., the $26-\mathrm{cm}$ stick). With the candy $16 \mathrm{~cm}$ from the open end of the tube, the 8 - and $12-\mathrm{cm}$ sticks were replaced with $16-$ and 20 -cm sticks. We used the 12- and 20-cm sticks because Experiment 1 showed that the mean working length of the sticks selected was approximately $3.5 \mathrm{~cm}$ less than their actual lengths. The lengths of the sticks whose working lengths best matched the distance over which the candy had to be retrieved were 12 and $20 \mathrm{~cm}$ for the 8 - and $16-\mathrm{cm}$ distances, respectively.

If the participants' selections in Experiment 1 reflected their attempts to match the working length of a stick to the candy's distance (coupled with judgment error), simplifying the choice to two alternatives should reduce this error and participants should then select the 12- and $20-\mathrm{cm}$ sticks when available. However, if there is any tendency to match a stick's actual length to an object's distance, the participants should select the 8 - and $16-\mathrm{cm}$ sticks when available. Finally, if the preferred strategy is to select the longest stick in the pair, the participants will do so, regardless of the other stick's actual or working length. The results of Experiment 1 suggest that these latter two outcomes are unlikely.

\section{Method}

Participants. Eighty-three undergraduate students attending a liberal arts college in southern California volunteered for the study and received partial course credit for their involvement.

Materials and Procedure. The same general procedure from Experiment 1 was used here, with the following exceptions: With the candy $8 \mathrm{~cm}$ from the open end of the tube, Group 8-26 $(n=12)$ selected an 8- or a 26 -cm stick to retrieve the candy; Group 8-12 $(n=12)$ selected an 8- or a 12-cm stick; and Group 12-26 $(n=15)$ selected a $12-$ or a $26-\mathrm{cm}$ stick. With the candy $16 \mathrm{~cm}$ from the open end of the tube, Group 16-26 $(n=15)$ selected a 16- or a $26-\mathrm{cm}$ stick to retrieve the candy; Group 16-20 $(n=14)$ selected a 16 - or a $20-\mathrm{cm}$ stick; and Group 20-26 $(n=15)$ selected a 20 - or a $26-\mathrm{cm}$ stick.

\section{Results and Discussion}

Figure 3 shows how the participants distributed their selections when the candy was $8 \mathrm{~cm}$ from the open end of the tube. All 12 people in Group 8-26 (top panel) selected

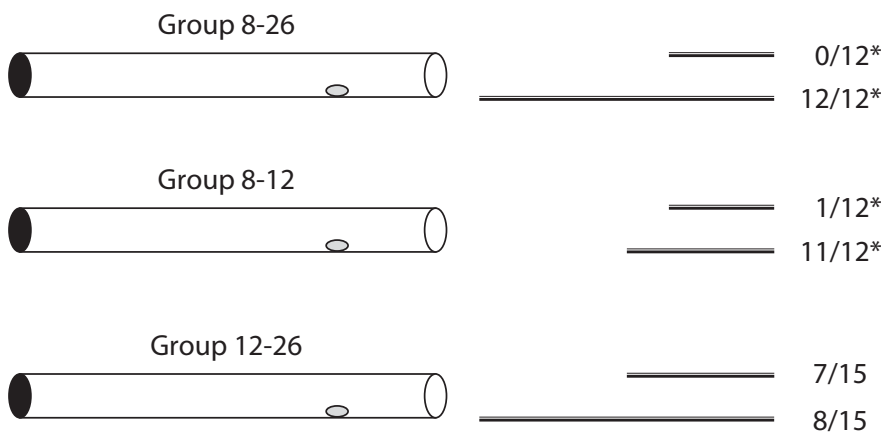

Figure 3. The two-alternative stimulus arrangements used in Experiment 2 when the candy was $8 \mathrm{~cm}$ from the open end of the tube (not drawn to scale). The long and short sticks next to a tube represent the longer and shorter sticks used in that configuration. The data next to each stick show the number of people who selected that stick. An asterisk next to the result indicates statistical significance. 


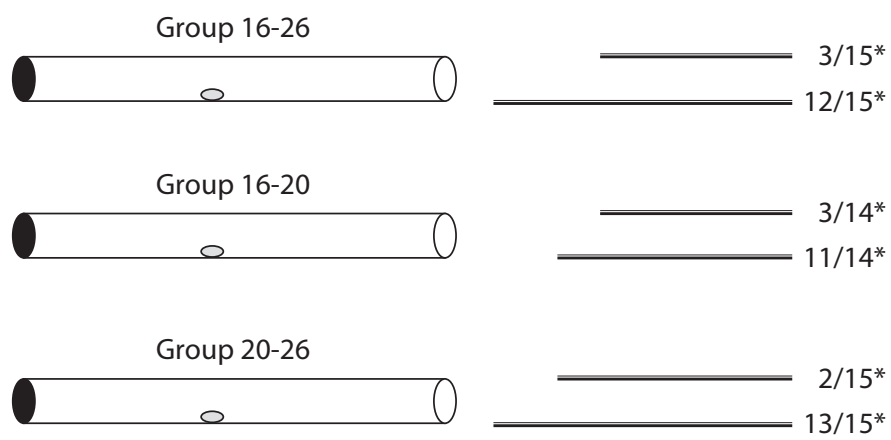

Figure 4. The two-alternative stimulus arrangements used in Experiment 2 when the candy was $16 \mathrm{~cm}$ from the open end of the tube (not drawn to scale). Details are the same as in Figure 3.

the 26-cm stick, a result that differs significantly from that expected by chance [binomial parameters: $n=12 ; k=12$; $p=.5 ; P$ of 12 or more out of $12<.01]$. Eleven of the 12 participants in Group 8-12 (middle panel) selected the $12-\mathrm{cm}$ stick, a result that also differs significantly from random responding [binomial parameters: $n=12 ; k=$ $11 ; p=.5 ; P$ of 11 or more out of $12<.01]$. Seven of the 15 participants in Group 12-26 (bottom panel) selected the $12-\mathrm{cm}$ stick, and 8 of the participants selected the $26-\mathrm{cm}$ stick, outcomes that would be expected by chance [binomial parameters: $n=15 ; k=8 ; p=.5 ; P$ of 8 or more out of $15=.50]$.

Figure 4 shows how the participants distributed their selections when the candy was $16 \mathrm{~cm}$ from the open end of the tube. Twelve of the 15 people in Group 16-26 selected the 26-cm stick, a result that differs significantly from that expected by chance [binomial parameters: $n=15 ; k=12$; $p=.5 ; P$ of 12 or more out of $15=.02]$. Eleven of the 14 participants in Group 16-20 selected the 20 -cm stick, a result that also differs significantly from random responding [binomial parameters: $n=14 ; k=11 ; p=.5 ; P$ of 11 or more out of $14=.02]$. Thirteen of the 15 participants in Group 20-26 selected the 26-cm stick, an outcome that differs significantly from that expected by chance [binomial parameters: $n=15 ; k=13 ; p=.5 ; P$ of 13 or more out of $15<.01]$.

The results showed that the participants had no bias to select a tool whose working length matched the distance over which an object had to be retrieved; had they had such a bias, they would have selected the $12-$ and $20-\mathrm{cm}$ sticks when available. A preponderance of participants did not select these sticks. Thus, it does not seem that the participants' selections in Experiment 1 reflected erroneous attempts to match the working length of a stick to the candy's distance.

For the participants in Group 12-26, however, the absence of a preference for either the $12-$ or $26-\mathrm{cm}$ sticks does not mean that the participants' selections were uncontrolled or random. It is more likely that different people's selections were controlled by different factors. Evidence for this conclusion comes from the participants' explanations for their selection. People who selected the $12-\mathrm{cm}$ stick said things related to the tool's usability (e.g., "It was shorter and easier to maneuver"; or, "It was smaller and I would have more control"). In contrast, people who selected the $26-\mathrm{cm}$ stick said things related to adaptability (e.g., "If I pushed the M\&M back, the longer stick could get it"; or, "I could get deeper into the tube in case the M\&M goes back a bit").

In contrast to Group 12-26, the participants in Group 20-26 preferred the longer stick. The difference between these two groups' selections is puzzling. As the two sticks become more similar in length, people may select the longer stick because there is no significant downside to selecting it. Recall that people in Group 12-26 who selected the $12-\mathrm{cm}$ stick said they thought this shorter stick would be easier to use. The 2 people in Group 20-26 who selected the $20-\mathrm{cm}$ stick also explained their selection by referring to the shorter stick's ease of use (e.g., "It [the reward] was easier to drag [with this stick]"; or, "Easier to maneuver"). These sorts of explanations suggest that, for some people, there is a cost to using an unnecessarily long stick. This cost diminishes as the sticks become similar; under these circumstances, people prefer the longer stick. In short, it seems that people's tool selections depend on an interaction between the distance over which a desired object has to be retrieved and the available tools.

\section{EXPERIMENT 3}

We sought to examine in Experiment 3 the generality of Experiment 2's results and whether the comparison stimuli influenced people's tool selections. To examine these issues, we used stimulus arrangements that pitted the key sticks from the previous experiments against each other within a single arrangement. With the candy $8 \mathrm{~cm}$ from the open end of the tube, a group of participants selected from among 8-, 12-, and 26-cm sticks. With the candy $16 \mathrm{~cm}$ from the open end of the tube, a second group of participants selected from among 16-, 20-, and 26-cm sticks. With the candy $8 \mathrm{~cm}$ from the open end of the tube, a third group of participants selected from among 12-, $20-$, and 26-cm sticks.

If the set of tools has no effect on participants' tool selections, their selections should be similar to those observed in Experiment 2. That is, the participants in Group 8-12-26 
should select equally the 12 - and 26-cm sticks and should not select the 8-cm stick; the people in Group 16-20-26 should prefer the $26-\mathrm{cm}$ stick and should not select the $16-\mathrm{cm}$ stick, although a small and statistically insignificant proportion of people might select the $20-\mathrm{cm}$ stick; and Group 12-20-26's selections should be the same as those of Group 8-12-26 because the candy's distance is the same for both of these groups. The only difference between these groups was the third alternative (an 8-cm stick for one group and a $20-\mathrm{cm}$ stick for the other).

If the set of tools influences which tool people select, predicting the outcomes is difficult because there is no clear theory or data to suggest what will happen. Nevertheless, there is reason to think that the set of tools will affect the tool that people select. Research in psychophysics suggests that perceptual judgments are influenced by the context in which stimuli are evaluated. The absolute magnitude of a stimulus is judged to be smaller when it is presented with similar but smaller stimuli than when it is presented along with similar but larger stimuli (Parducci, 1968). Two well-known examples of this phenomenon are the Delboeuf Illusion and the Ebbinghaus Effect, the latter being an illusion that is produced by ringing a circle of a particular size with either larger or smaller circles. The circle in the center is perceived as being larger when it is ringed by smaller circles than when it is surrounded by larger circles (see Burton, 2001). Also, psychophysicists have long known that judgments tend to be pulled toward the center of the range (e.g., Hollingworth, 1909; Reynolds \& Stevens, 1960). And cognitive and social psychologists tell us that people's judgments are influenced by stimuli that anchor and frame alternatives (Tversky \& Kahneman, 1974), even when the anchor is irrelevant to the task (Wilson, Houston, Etling, \& Brekke, 1996).

\section{Method}

Participants. Forty-six undergraduate students attending a liberal arts college in southern California volunteered for the study and received partial course credit for their involvement.

Materials and Procedure. The same materials, general setup, and procedure from Experiment 2 were used here with the follow- ing exceptions: Group 8-12-26 $(n=12)$ selected from among the $8-, 12-$, and $26-\mathrm{cm}$ sticks to retrieve the candy that was $8 \mathrm{~cm}$ from the open end of the tube; Group 16-20-26 $(n=20)$ selected from among the 16-, 20-, and 26-cm sticks to retrieve the candy that was $16 \mathrm{~cm}$ from the open end of the tube; and Group 12-20-26 $(n=14)$ selected from among the 12-, 20-, and 26-cm sticks to retrieve the candy when it was $8 \mathrm{~cm}$ from the open end of the tube. To protect against Type I error inflation related to multiple comparisons, we adjusted $\alpha$ using Keppel's modified Bonferroni correction (Keppel, 1991). This yielded $\alpha_{\text {new }}<.04754$ for all post hoc chi-square tests. However, for simplicity, and because this correction made data analyses appropriately more conservative in situations where the expected frequency for a category was less than 5 , we used $\alpha_{\text {new }}$ for all analyses in Experiment 3.

\section{Results and Discussion}

Figure 5 shows how the participants distributed their selections. The top panel shows that no one in Group 8-1226 selected the 8 -cm stick, 9 of the 12 participants selected the $12-\mathrm{cm}$ stick, and 3 of the 12 participants selected the $26-\mathrm{cm}$ stick. This distribution of selections is statistically significant $\left[\chi^{2}(2)=10.50, p<.01\right]$. Post hoc chi-square tests showed that the number of people who selected the 8and $12-\mathrm{cm}$ sticks differed significantly from what would be expected by chance $\left[\chi^{2} \mathrm{~s}(1)>4.59, p \mathrm{~s}<.03\right]$ but that the number of people who selected the 26 -cm stick did not $\left[\chi^{2}(1)=0.09, p=.76\right]$.

The middle panel shows that 1 person in Group 16-20-26 selected the $16-\mathrm{cm}$ stick, 7 of the 20 participants selected the 20 -cm stick, and 12 of the 20 participants selected the $26-\mathrm{cm}$ stick. This distribution of selections is statistically significant $\left[\chi^{2}(2)=9.10, p=.01\right]$. Post hoc chi-square tests showed that the number of people who selected the $16-$ and $26-\mathrm{cm}$ sticks differed significantly from what would be expected by chance $\left[\chi^{2} \mathrm{~s}(1)>5.25, p \mathrm{~s}<.03\right]$ but that the number of people who selected the $20-\mathrm{cm}$ stick did not $\left[\chi^{2}(1)=0.00, p=1\right]$.

The bottom panel shows how the participants in Group 12-20-26 distributed their selections. As with Group 8-12-26, the candy was $8 \mathrm{~cm}$ from the open end of the tube. The results showed that 4 of the 14 participants selected the $12-\mathrm{cm}$ stick, 9 people selected the $20-\mathrm{cm}$

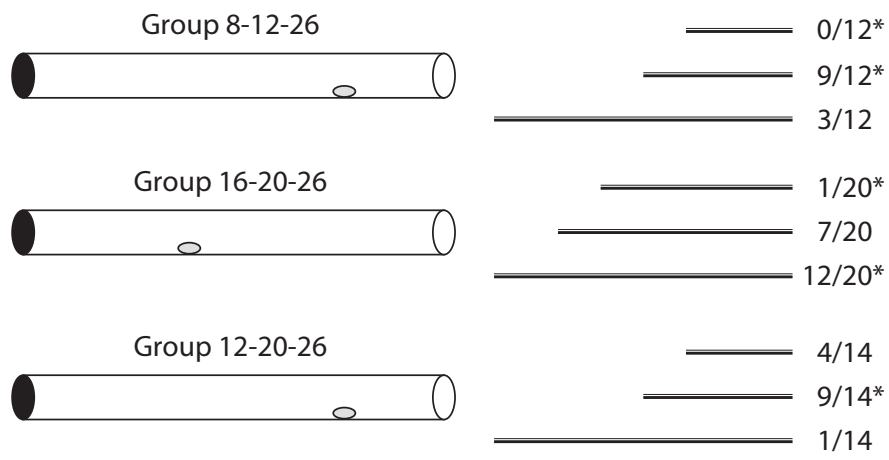

Figure 5. The three-alternative stimulus arrangements used in Experiment 3 (not drawn to scale). For Groups 8-12-26 and 12-20-26, the candy was $8 \mathrm{~cm}$ from the open end of the tube. For Group 16-20-26, the candy was $16 \mathrm{~cm}$ from the open end of the tube. The sticks next to a tube represent the different stick lengths used in that configuration. Details are the same as in Figure 3. 
stick, and 1 person chose the 26 -cm stick. This distribution of selections is statistically significant $\left[\chi^{2}(2)=\right.$ $7.00, p=.03]$. Post hoc chi-square tests showed that the number of people who selected the 20-cm stick differed significantly from what would be expected by chance $\left[\chi^{2}(1)=4.72, p=.03\right]$ but that the number of people who selected the 12- and $26-\mathrm{cm}$ sticks did not $\left[\chi^{2} \mathrm{~s}(1)<\right.$ $3.22, p \mathrm{~s}>.07]$.

These results replicated those of Experiments 1 and 2 by showing again that the participants did not have a tendency to match a stick's length to an object's distance. If the participants had this tendency, they would have selected the 8- and 16-cm sticks. They did not. Also, in a replication of a result from Experiment 2 (Group 20-26), people preferred the longest stick when the difference between it and a stick whose working length matched the object's distance was relatively short (Group 16-20-26).

More importantly, the results showed that whether someone selected a stick with a working length that matched an object's distance or a longer stick depended on the alternatives. The participants preferred the stick whose working length matched the object's distance (i.e., the $12-\mathrm{cm}$ stick) when the alternatives were sticks $4 \mathrm{~cm}$ shorter and $14 \mathrm{~cm}$ longer (Group 8-12-26). However, the participants preferred a 20 -cm stick-a stick $8 \mathrm{~cm}$ longer than one whose working length matched an object's distance-when it was pitted against sticks $8 \mathrm{~cm}$ shorter and $6 \mathrm{~cm}$ longer (Group 12-20-26). There is no simple explanation for these results. For example, it is unlikely that participants simply preferred the middle-sized stick. If this were the case, the people in Group 16-20-26 would have preferred the $20-\mathrm{cm}$ stick. The best we can say is that the participants' selections may have been influenced by the anchors (see Petrov \& Anderson, 2005), uncertainty that led to the central judgment effect (see Matthews \& Stewart, 2009), differences in the magnitude of adjacent stimuli (see Brown, Marley, Dodds, \& Heathcote, 2009), or some other unspecified comparison among the alternatives. Without a large-scale parametric investigation that varied the anchors, magnitude of the alternatives, number of alternatives, spacing of the differences in magnitude between alternatives, and the distance over which the candy had to be retrieved, we cannot say what explains the difference between Groups 8-12-26 and 12-20-26. However, we can speculate as to what contributed to this difference. We will return to this point below.

\section{GENERAL DISCUSSION}

The present study addressed the need, noted by Bluff et al. (2007), for a comparison between corvids' tool selections and those of humans and other primates. This study showed that, unlike some New Caledonian crows tested with similar tasks (see, e.g., Chappell \& Kacelnik, 2002), people did not generally select a stick tool whose length matched the distance over which a desired object had to be retrieved. If there was any tendency to match stick length to object distance, the matching was situational and between a stick's working, not actual, length, and the object's distance. There was little evidence that people generally (i.e., across both distances over which the candy had to be retrieved and across all stimulus arrangements) matched a stick's working length to the object's distance. This study also showed that, unlike some crows, people do not have a preference for the longest stick in a set.

This absence of matching of a stick's length to an object's distance is perhaps unsurprising. Hunt et al. (2006) and Tebbich and Bshary (2004) argued that this would not be an efficient or generalizable strategy for tool-using birds such as New Caledonian crows and woodpecker finches (see the beginning of this article). To their argument, we add the following: Perhaps Chappell and Kacelnik's (2002) crows' tendency to match a stick's length to an object's distance was a statistical anomaly. To illustrate, consider the following results reported by Chappell and Kacelnik (in their Experiment 1). On 10 of 20 trials, Bird A selected the longest stick in the 10-stick set; on 1 of these trials, though, the longest stick was confounded with the farthest distance over which the food had to be retrieved. On 5 of 20 trials, this bird selected a stick whose length matched the distance of the food from the open end of the tube; but, again, the matching stick was also the longest stick on 1 trial. On 5 of 20 trials, this bird selected a stick long enough to retrieve food, but which was neither the longest stick nor a stick whose length matched the distance over which the food had to be retrieved. On 1 of 20 trials, this bird selected a stick that was too short to retrieve the food.

If we exclude the confounded matching distancethat is, the distance that also corresponded to the longest stick in the set-then Bird A selected the longest stick on 9 of 19 trials, a proportion that exceeds that expected by chance, and chose the stick that matched the food's distance on 4 of 19 trials, a proportion consistent with random responding. The second crow (Bird B) behaved identically to Bird A in terms of selecting the longest stick or the matching stick. Although there is no doubt that the crows selected the longest stick more often than expected by chance, they may not have preferred a stick whose length matched the food's distance. At the very least, the propensity to select a matching stick seems weak.

Although our participants' tool selections were different from those made by Chappell and Kacelnik's (2002) crows, their selections were related to the distance over which the desired object had to be retrieved. This finding contrasts with Hunt et al.'s (2006) results that there was no relationship between the length of leaf- and twig-tools selected by New Caledonian crows and the depth of the holes that contained the food. In relation to previous research with avian species, the results of the present study most closely resemble those observed with woodpecker finches. These birds generally selected sticks that were longer than necessary, though not always the longest stick in a set, to retrieve food (Tebbich \& Bshary, 2004). Finally, this study showed that people's tool selections depend on the stimulus context. Different proportions of people sometimes preferred (or avoided) the same tools, depending on the number and lengths of the alternative tools.

Given the constellation of results, what controlled the participants' tool selections? We believe that their selections accord with the following account. 

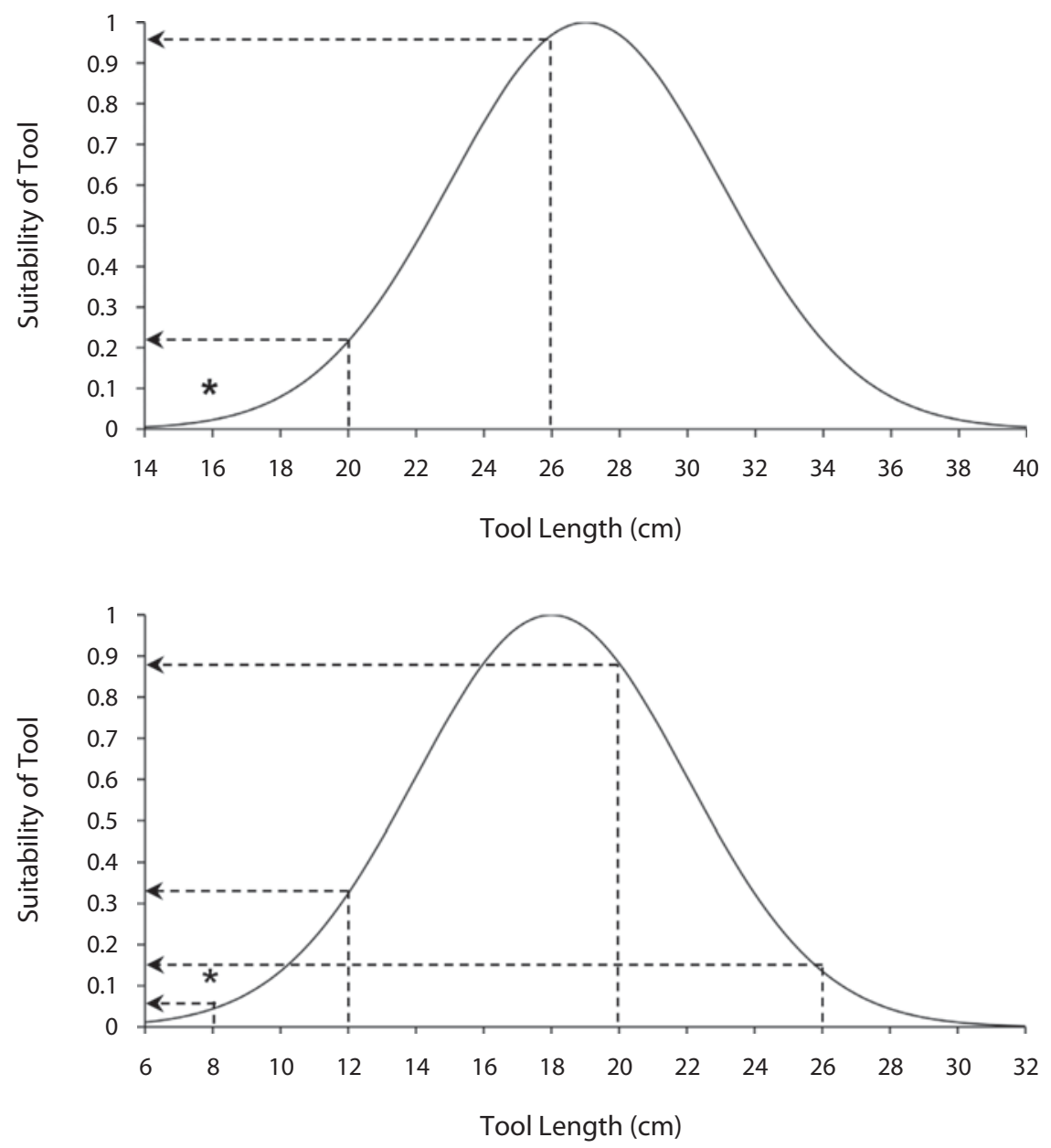

Figure 6. Hypothetical psychometric functions relating the suitability of a tool to its length. The top graph shows the function for when the desired object (illustrated by the asterisk) is $16 \mathrm{~cm}$ from the open end of the tube, and the 20 - or $26-\mathrm{cm}$ sticks can be selected to retrieve the object. This graph shows that, given the stimulus configuration (including the size and shape of the tube), the optimal stick length is about $27 \mathrm{~cm}$. As such, people should generally prefer the $26-\mathrm{cm}$ tool when the alternative is a $20-\mathrm{cm}$ tool. The bottom graph shows the function when the desired object is $8 \mathrm{~cm}$ from the open end of the tube and people have to select from among the 8-, 12-, and 26-cm sticks or the 12-, 20-, and 26-cm sticks. For the former arrangement, most people should select the 12-cm stick; for the latter arrangement, most people should select the 20 -cm stick.

1. For any distance over which an object must be retrieved, there is a tool of optimal length to retrieve it that depends on the physical characteristics of the tools and the task, and on how someone uses the tools. For example, if an object is $8 \mathrm{~cm}$ from the open end of a tube, some tools are too short to reach the object (e.g., 4-cm stick), some tools can reach the object if the participant holds them in a particular way and can partially insert fingers or beak into the tube (e.g., 8-cm stick), and some tools can reach the object but may be awkward or unwieldy to use given the objective and the environment (e.g., 26-cm stick).

2. The problem is that, because the participants had no prior experience with the tools or the stimulus arrangements, there was some degree of error in their judgment of the distance over which an object had to be retrieved, a tool's length, and how they would use the tool.
3. Moreover, this judgment was influenced by the context. Just as a square of a particular size is judged as being small when presented alongside larger squares but judged as being large when presented alongside smaller squares, the perceived length (and thus suitability) of a stick was influenced by the lengths of the alternative stimuli.

A schematic of this account is presented in Figure 6. The graphs depict how a tool's suitability to retrieve an object varies as a function of its length. The characteristics of a curve depend on the set of tools from which a tool is selected and the amount of judgment error (which is itself partly a function of the tools in the set). The more suitable a tool, the more likely it will be selected. For example, the curve of the top graph is consistent with Group 20-26's tool selections in Experiment 2 when there were two alternatives and the candy was $16 \mathrm{~cm}$ away; the curve in 
the bottom graph is consistent with people's selections in Experiment 3, when the candy was $8 \mathrm{~cm}$ from the open end of the tube and the alternatives consisted of 8-, 12-, and 26-cm sticks or 12-, 20-, and 26-cm sticks.

If there is any truth to this account, the results highlight two important implications for the study of physical cognition, one methodological and the other conceptual. Methodologically, the results suggest that a relatively small change in a task might cause an animal to behave like a human and vice versa (e.g., Mulcahy \& Call, 2006; Seed, Call, Emery, \& Clayton, 2009; Silva et al., 2008). Conceptually, the results suggest that researchers studying physical cognition across species need to be cautious about designing experiments that seemingly are critical tests of competing hypotheses, such as Povinelli's (2000) tests of stimulus-bound perceptual understanding versus generalizable abstract reasoning in chimpanzees (Machado \& Silva, 2003). As others have cautioned, the task itself is not trivial or theoretically unimportant (Timberlake, 2001, 2002). A chimpanzee that cannot reliably use a stick to push a cookie out of a clear tube may be doing so not because of its physical cognition, but because this task interferes with a species-typical behavior related to procuring food (cf. Breland \& Breland, 1961). What researchers need to do is identify the environmental conditions that control different tool-use responses (Machado \& Silva, 2003; Tomasello \& Call, 1997). In relation to the tasks used in the present study, crows do not appear to be "feathered humans" (cf. Emery, 2004). It remains for future research to explore more completely how the stimulus context and learning affect humans' and crows' tool selections and to determine the conditions that cause birds to behave like feathered apes or people to behave like featherless crows.

\section{AUTHOR NOTE}

We thank Bill Timberlake for helpful suggestions and ideas for follow-up experiments, and Armando Machado for helpful comments and discussions, especially regarding the statistical analyses. Address correspondence to F. J. or K. M. Silva, Department of Psychology, P.O. Box 3080, 1200 East Colton Avenue, Redlands, California 92373-0999 (e-mail: francisco_silva@redlands.edu or kathleen_silva@redlands.edu).

\section{REFERENCES}

Bluff, L. A., Weir, A. A. S., Rutz, C., Wimpenny, J. H., \& KacelNIK, A. (2007). Tool-related cognition in New Caledonian crows. Comparative Cognition \& Behavior Reviews, 2, 1-25.

Breland, K., \& Breland, M. (1961). The misbehavior of organisms. American Psychologist, 16, 681-684.

Brown, S. D., Marley, A. A. J., Dodds, P., \& Heathcote, A. (2009). Purely relative models cannot provide a general account of absolute identification. Psychonomic Bulletin \& Review, 16, 583-593. doi:10.3758/PBR.16.3.583

Burton, G. (2001). The tenacity of historical misinformation: Titchener did not invent the Titchener illusion. History of Psychology, 4, 228-244.

Chappell, J., \& KaCELniK, A. (2002). Tool selectivity in a non-primate, the New Caledonian crow (Corvus moneduloides). Animal Cognition, 5, 71-78. doi:10.1007/s10071-002-0130-2

EMERY, N. J. (2004). Are corvids "feathered apes"? Cognitive evolution in crows, jays, rooks, and jackdaws. In S. Watanabe (Ed.), Comparative analysis of minds (pp. 181-213). Tokyo: Keio University Press.

Emery, N. J., \& Clayton, N. S. (2009). Tool use and physical cognition in birds and mammals. Current Opinion in Neurobiology, 19, 27-33. doi:10.1016/j.conb.2009.02.003

HollingworTH, H. L. (1909). The inaccuracy of movement. New York: Science Press.

Hunt, G. R., Rutledge, R. B., \& Gray, R. D. (2006). The right tool for the job: What strategies do wild New Caledonian crows use? Animal Cognition, 9, 307-316. doi:10.1007/s10071-006-0047-2

KePPEL, G. (1991). Design and analysis: A researcher's handbook (3rd ed.). Upper Saddle River, NJ: Prentice Hall.

Machado, A., \& Silva, F. J. (2003). You can lead an ape to a tool, but ... . : A review of Povinelli's Folk physics for apes: The chimpanzee's theory of how the world works. Journal of the Experimental Analysis of Behavior, 79, 267-286. doi:10.1901/jeab.2003.79-267

Matthews, W. J., \& Stewart, N. (2009). Psychophysics and the judgment of price: Judging complex objects on a non-physical dimension elicits sequential effects like those in perceptual tasks. Judgment \& Decision Making, 4, 64-81.

Mulcahy, N. J., \& Call, J. (2006). How great apes perform on a modified trap-tube task. Animal Cognition, 9, 193-199. doi:10.1007/ s10071-006-0019-6

PARDuccI, A. (1968). The relativism of absolute judgment. Scientific American, 219, 84-90.

Petrov, A. A., \& Anderson, J. R. (2005). The dynamics of scaling: A memory-based anchor model of category rating and absolute identification. Psychological Review, 112, 383-416. doi:10.3758/ PBR.16.3.594

Povinelli, D. J. (2000). Folk physics for apes: The chimpanzee's theory of how the world works. Oxford: Oxford University Press.

REynolds, G. S., \& Stevens, S. S. (1960). Binaural summation of loudness. Journal of the Acoustical Society of America, 32, 1337-1344.

Seed, A. M., Call, J., Emery, N. J., \& Clayton, N. S. (2009). Chimpanzees solve the trap problem when the confound of tool-use is removed. Journal of Experimental Psychology: Animal Behavior Processes, 35, 23-34. doi:10.1037/a0012925

Silva, F. J., Page, D. M., \& Silva, K. M. (2005). Methodologicalconceptual problems in the study of chimpanzees' folk physics: How studies with adult humans can help. Learning \& Behavior, 33, 47-58.

Silva, F. J., Silva, K. M., Cover, K. R., Leslie, A. L., \& Rubalcaba, M. A. (2008). Humans' folk physics is sensitive to physical connection and contact between a tool and reward. Behavioural Processes, 77, 327-333. doi:10.1016/j.beproc.2007.08.001

Tebbich, S., \& Bshary, R. (2004). Cognitive abilities related to tool use in the woodpecker finch, Cactospiza pallida. Animal Behaviour, 67, 689-697. doi:10.1016/j.anbehav.2003.08.003

Timberlake, W. (2001). Integrating niche-related and general process approaches in the study of learning. Behavioural Processes, 54, 7994. doi:10.1016/S0376-6357(01)00151-6

TimberlaKe, W. (2002). Niche-related learning in laboratory paradigms: The case of maze behavior in laboratory rats. Behavioural Brain Research, 134, 355-374. doi:10.1016/S0166-4328(02)00048-7

Tomasello, M., \& Call, J. (1997). Primate cognition. New York: Oxford University Press.

Tversky, A., \& Kahneman, D. (1974). Judgment under uncertainty: Heuristics and biases. Science, 185, 1124-1131.

Wilson, T. D., Houston, C. E., Etling, K. M., \& Brekke, N. (1996). A new look at anchoring effects: Basic anchoring and its antecedents. Journal of Experimental Psychology: General, 125, 387-402.

(Manuscript received May 29, 2009;

revision accepted for publication September 5, 2009.) 